\title{
Correspondence
}

\section{Accuracy of routine clinical test weighing}

\section{Sir,}

I congratulate Whitfield et al. ${ }^{1}$ on their thorough analysis of the accuracy of test feeding in the clinical situation, but disagree with their statement that 'test weighing with clinical baby scales is an unreliable and inaccurate indication of feed intake .... They used Salter 'Trent' scales; these are spring balances with the scale marked in $10 \mathrm{~g}$ divisions and variations of up to $10 \mathrm{~g}$ due to parallax must be expected; therefore this type of balance is unsuitable for this purpose.

Beam-balances, for example those manufactured by Ohio Limited and Stevens and Son, are popular choices for clinical use. These avoid such errors and their accuracy can be enhanced by adding 1 to $5 \mathrm{~g}$ weights to the tray.

When using such a system Rahilly ${ }^{2}$ reported a correlation coefficient between the volume of milk given and the consequent weight gain in 17 babies of 0.998 . The measurements were carefully made by a nurse without special training. Many highly trained nurses are excellent cooks and I cannot believe that 'the pressures of modern technology' have impaired their ability to weigh accurately if given the appropriate equipment.

Test weighing can be useful during the establishment of breast feeding and I think it would be wrong to allow its 'general' dismissal to pass without comment.

\section{References}

1 Whitfield M F, Kay R, Stevens S. Validity of routine clinical test weighing as a measure of the intake of breast-fed infants. Arch Dis Child 1981 ; 56: 919-21.

2 Rahilly P M. Effects of sleep state and feeding on cranial blood flow of the human neonate. Arch Dis Child 1980; 55: $265-70$.

J K STOTHERS Joint Academic Department of Child Health,

London Hospital Medical College, Turner Street, London E1 $2 A D$

Dr Whitfield comments:

I must stress that the purpose of our study was to make an assessment of the magnitude of the errors likely to occur in routine clinical test weighing, to assist paediatricians in the interpretation of test weighing results presented to them. The study was conducted under clinical 'field' conditions using the type of baby scales routinely available in our hospital, several observers, and several sets of scales. Apart from periodic maintenance and calibration carried out by the hospital for clinical reasons, no additional check on the accuracy of individual scales was made.
Under these conditions we found that test weighing gave an 'unreliable indication of feed intake in breastfed infants'. A better result was obtained by Culley $\boldsymbol{e} t$ al. ${ }^{1}$, although considerable scatter was evident even when observer variability was excluded. The use of a beam balance would provide greater accuracy with the motionless infant; the same degree of accuracy should not be expected when weighing a restless, hungry infant.

The contributions to the total error made by movement of the baby, observer error, and scale inaccuracies, can be minimised by the use of an electronic balance which takes a series of measurements in quick succession. ${ }^{2}$

In the neonatal unit, the ability to measure an infant's weight precisely is important, not only to minimise inaccuracies in test weighing, but also to ascertain adequate weight growth. Acquisition of at least one set of highly accurate scales for this purpose should be considered a budgetary priority.

\section{References}

1 Culley P, Milan P, Roginski C, Waterhouse J, Wood B. Are breast-fed babies still getting a raw deal in hospital? Br Med J 1979; ii: 891-3.

2 Lucas A, Lucas P J, Baum J D. Pattern of milk flow in breast-fed infants. Lancet 1979; ii : 57-8.

Sir,

Whitfield $e t$ al. concluded that the use of test weighing to measure milk intake in special care babies gives results that are not only inaccurate but also biased, particularly at the extremes. The charge of inaccuracy is true because the usual weighing scales are difficult to read to better than $20 \mathrm{~g}$. For babies with only a small intake per feed this uncertainty is sufficient to affect the measurement appreciably. For older or more healthy babies, where the milk volume per feed is greater, the measurement error is less important. In either case the accuracy can be greatly improved by weighing with an electronic balance which records the baby's weight to within $1 \mathrm{~g}$.

The suggestion that test weighing gives a biased result is more serious. They looked at the regression of feed weight (or FW) on test weight (TW), where FW is the accurately known change in weight of the bottle over the feed, and TW is the observed test weight. This regression has a slope of $0 \cdot 40$, significantly less than $1(P<0 \cdot 001)$. In general it is unwise to compare two methods of measurement using the regression of one on the other, as neither $Y$ regressed on $X$ nor $X$ on $Y$ gives the correct answer. However, in the present case where FW is measured virtually without error, it is correct to use regression, but with FW as the independent variable. The regression of TW on FW gives a slope of 0.78 with standard error $\mathbf{0 . 1 2}$, which is insignificantly different from $1(t=1 \cdot 81, \mathrm{P}>0 \cdot 05)$. Thus the suggestion of bias 